\title{
IMPACT OF NOTCH SIGNALING ON THE PROGNOSIS OF PATIENTS WITH HEAD AND NECK SQUAMOUS CELL CARCINOMA
}

Gianluigi Grilli ${ }^{1}$, Francisco Hermida-Prado ${ }^{2,3,4}$, Mónica Álvarez-Fernández ${ }^{2,3}$, Eva Allonca ${ }^{2,3,4}$, Miguel Álvarez-González ${ }^{2,3}$, Aurora Astudillo³,5, Gema Moreno-Bueno ${ }^{4,6,7}$, Amparo Cano ${ }^{4,6}$, Juana M. García-Pedrero ${ }^{2,3,4 *}$, Juan Pablo Rodrigo ${ }^{2,3,4 *}$.

${ }^{1}$ Department of Otolaryngology, Ospedali Riuniti and Università degli Studi di Foggia, Foggia, Italy.

${ }^{2}$ Department of Otolaryngology, Hospital Universitario Central de Asturias, Instituto de Investigación Sanitaria del Principado de Asturias, Oviedo, Spain.

${ }^{3}$ Instituto Universitario de Oncología del Principado de Asturias, University of Oviedo, Oviedo, Spain.

${ }^{4}$ Centro de Investigación Biomédica en Red de Cáncer (CIBERONC), Instituto de Salud Carlos III, Madrid, Spain.

${ }^{5}$ Departamento de Patología, Hospital Universitario Central de Asturias, ISPA, Oviedo, Spain.

${ }^{6}$ Departamento de Bioquímica, Universidad Autónoma de Madrid (UAM), Instituto de Investigaciones Biomédicas 'Alberto Sols' (CSIC-UAM), IdiPAZ, Madrid, Spain.

${ }^{7}$ Fundación MD Anderson Internacional Madrid, Spain.

\section{* Correspondence:}

Juan P. Rodrigo, MD, PhD E-mail: juanpablo.rodrigo@sespa.es

Juana M. García-Pedrero, PhD E-mail: juanagp.finba@gmail.com

Hospital Universitario Central de Asturias

Edificio FINBA, Lab ORL

33011 Oviedo, Spain

Word Count: 3492 



\section{DISCLOSURE OF POTENTIAL CONFLICTS OF INTEREST}

No potential conflicts of interest were disclosed. 


\section{ABSTRACT}

Objectives: The function of NOTCH signaling (oncogenic or oncosuppressive) remains controversial in head and neck squamous cell carcinomas (HNSCC). The purpose of this work is to investigate the role of $\mathrm{NOTCH}$ pathway in HNSCC prognosis.

Methods: immunohistochemical NOTCH1 and HES1 expression was jointly evaluated and correlated with other NOTCH1 targets, p21(WAF1/Cip1) and Cyclin D1, using an unbiased cohort of 372 surgically treated HPV-negative HNSCC patients.

Results: Membranous NOTCH1 expression was detected in $197(61 \%)$ out of 324 evaluable tumor samples, and nuclear NOTCH1 expression in 91 samples (28\%). Nuclear HES1 expression was found in 224 (67\%) cases. Membranous and nuclear $\mathrm{NOTCH} 1$ expression were consistently and significantly correlated with nuclear HES1 $(\mathrm{P}<0.001)$ and $\mathrm{p} 21(\mathrm{P}<0.05)$ expression, but not with Cyclin D1. NOTCH1 expression was significantly associated to early stages (I-II), non-recurrent disease, and better disease-specific (DSS) and overall survival (OS) rates $(P<0.001)$. Moreover, triplepositive cases (NOTCH1+/HES1+/p21+) exhibited significantly improved DSS $(P<0.001)$ and OS $(P=0.004)$, thus reinforcing the association of $\mathrm{NOTCH}$ pathway activation with a better prognosis in HNSCC. Multivariate analysis further revealed membranous NOTCH1 expression as a robust independent predictor of better DSS $(\mathrm{HR}=0.554 ; 95 \%$ IC $0.412-0.745 ; \mathrm{P}<0.001)$ and better $\mathrm{OS}(\mathrm{HR}=0.640 ; 95 \% \mathrm{Cl} 0.491-$ $0.835 ; P=0.001)$.

Conclusion: These findings show the association of NOTCH pathway activation with a better prognosis in HNSCC patients, also revealing membranous NOTCH1 expression as a robust independent predictor of improved survival. Accordingly, these results suggest a tumor suppressive rather than an oncogenic role for $\mathrm{NOTCH}$ pathway in HNSCC.

Key words: NOTCH1, HES1, Head and neck squamous cell carcinoma, prognosis, survival 


\section{INTRODUCTION}

$\mathrm{NOTCH}$ proteins (NOTCH1-4 in humans) are a family of transmembrane receptors composed of an extracellular domain responsible for ligand recognition, a transmembrane domain, and an intracellular domain involved in signal transduction. Initiation of signaling is mediated through binding of the ligands Jagged (Jagged-1, Jagged-2) or Delta-like (DLL1, DLL3 and DLL4) resulting in the cleavage and release of the NOTCH intracellular domain (NICD), which is subsequently translocated to the nucleus, where it binds to the DNA-binding protein RBPJ (recombination signal binding protein for immunoglobulin-kappa-J region). This leads to the transcription of several target genes, including MYC and HES and HEY family proteins [1,2].

NOTCH signaling plays a fundamental role in various key biological processes, such as cell differentiation, proliferation, and self-renewal [3]. Alterations in NOTCH signaling have been described in different cancer types, which result in either tumor promotion or suppression depending on the tumor type and tissue context [1]. The primary function of NOTCH signaling in head and neck squamous cell carcinomas (HNSCC) remains controversial, since it may act as an oncogene, onco-suppressor, or even playing a bimodal action [4-6]. In-depth next generation sequencing (NGS) analysis of the HNSCC genome has revealed frequent mutations of the NOTCH receptor family, in particular NOTCH1 gene, most likely resulting in loss of function of the receptors $[7,8]$. Later, an integrated genomic analysis of $\mathrm{NOTCH}$ pathway that included gene expression, copy number alterations, point mutations and methylation, pointed this pathway as one of four major driver pathways in the pathogenesis of oral squamous cell carcinomas [9]. In that later study, NOTCH signaling pathway was found defective in $66 \%(23 / 35)$ of cases with alterations in several members of the pathway, including NOTCH1 and NOTCH2 mutations, copy number gains of JAG1, JAG2 and NUMB, and MAML1 and NOTCH2 losses [9].

To date, the clinical significance of the expression of NOTCH pathway components has 
only barely been studied using small HNSCC patient cohorts, and raising contradictory results $[2,10]$. Given the strong evidence pointing to a critical role for NOTCH signaling in HNSCC pathogenesis, efforts aimed at elucidating clinically relevant alterations of this pathway are fundamental to identify novel biomarkers as well as the key components to successfully develop targeted therapies. The aim of this study is to investigate the expression status of two main members of the pathway, $\mathrm{NOTCH} 1$ and HES1, together with the NOTCH1 targets p21(WAF1/Cip1/CDKN1A) and Cyclin D1 in a large cohort of surgically treated HPV-negative HNSCC patients to ascertain the prognostic role of NOTCH pathway activation in this cancer type.

\section{MATERIALS AND METHODS}

Patients and tissue specimens

Surgical tissue specimens from 382 patients who were diagnosed of HNSCC at the Hospital Universitario Central de Asturias between 1991 and 2010 were retrospectively collected, in accordance to approved institutional review board guidelines. All experimental protocols were approved by the Institutional Ethics Committee of the Hospital Universitario Central de Asturias and by the Regional CEIm from Principado de Asturias (date of approval May 14th, 2019; approval number: 141/19, for the project PI19/00560). Informed consent was obtained from all patients. All patients were surgically treated for a single primary tumor and received no treatment prior to surgery. No patient had distant metastasis at the time of diagnosis. Clinical, demographic and follow-up data were collected from the medical records. The tumors were staged according to the TNM system of the International Union Against Cancer (7th Edition).

Tissue sections were obtained from archival, formalin-fixed paraffin-embedded (FFPE) blocks provided by the Principado de Asturias BioBank (PT17/0015/0023), included into the Spanish National Biobanks Network. The histological diagnosis was confirmed by an experienced pathologist. Three 1-mm cylinders were taken from each FFPE 
tumor block to construct TMA blocks [11], containing a total of 249 oropharyngeal, 65 hypopharyngeal and 68 laryngeal SCC. In addition, each TMA included three cores of normal epithelium (pharyngeal and laryngeal mucosa obtained from non-oncologic patients).

Only 10 (3\%) HPV-positive tumors (8 oropharyngeal, 1 laryngeal and 1 hypopharyngeal) were detected using p16-immunohistochemistry, high-risk HPV DNA detection by in situ hybridization and genotyping by GP5+/6+-PCR, as previously reported $[11,12]$. Those cases were excluded from the analysis.

\section{Immunohistochemistry}

The TMAs were cut into $3-\mu \mathrm{m}$ sections and dried on Flex IHC microscope slides (Dako). The sections were deparaffinized with standard xylene and hydrated through graded alcohols into water. Antigen retrieval was performed using Envision Flex Target Retrieval solution, high $\mathrm{pH}$ (Dako). Staining was done at room temperature on an automatic staining workstation (Dako Autostainer Plus) using the anti-NOTCH1 monoclonal antibody (clone D1E11, Cell Signaling) at 1:400 dilution, the anti-HES1 monoclonal antibody (clone D6P2U, Cell Signaling) at 1:200 dilution, the anti-Cyclin D1 monoclonal antibody DCS-6 (Santa Cruz Biotechnology, Inc. sc-20044) at 1:100 dilution, and the anti-p21 monoclonal antibody (Clone 4D10; Leica Biosystems NCL-LWAF-1) at 1:10 dilution, for $30 \mathrm{~min}$. Immunodetection was carried out using the Dako EnVision Flex + Visualization System (Dako). Counterstaining with hematoxylin was the final step.

A semiquantitative scoring system based on staining intensity and the percentage of stained tumor cells was applied by two independent observers, blinded to clinical data. $\mathrm{NOTCH} 1$ and HES1 immunostaining was respectively scored from 0 to 2 if $0 \%$ to $10 \%$, $11 \%$ to $50 \%$, and $>50 \%$ of tumor cells showed membranous $\mathrm{NOTCH} 1$ staining or nuclear HES1 staining. The staining intensity was scored from 0 to 2 scale $(0=$ negative, 1 = weak, 2 = strong). The raw data were then converted to a 
Immunoreactive Score (IRS) by multiplying the quantity and staining intensity scores. Theoretically, the scores could range from 0 to 4 . For statistical purposes, these scores were dichotomized as negative expression (score 0) versus positive expression (scores 1-4). Since the NOTCH1 antibody recognizes both the full-length and the intracellular domain NICD, according to NOTCH1 function, staining into the nucleus was also separately evaluated as a surrogate of NOTCH1 activation. Nuclear NOTCH1 staining was scored in a binary fashion, as positive versus negative depending on the presence or absence of stained tumor cells. For p21 and Cyclin D1 proteins, nuclear staining was evaluated and dichotomized as negative expression (0-10\% stained cells) versus positive expression ( $>10 \%$ stained tumor cells). A high level of inter-observer concordance (>90\%) was achieved.

In silico analysis of NOTCH1 and HES1 mRNA expression using The Cancer Genome Atlas (TCGA) HNSCC database

mRNA expression analysis was performed using the transcriptome data from a TCGA cohort of 530 HNSCC patients [13]. mRNA levels of NOTCH1 and HES1 were compared between primary tumors $(n=520)$ and normal tissue samples $(n=44)$ using the UALCAN web tools (http://ualcan.path.uab.edu/) [14]. Correlations with clinicopathological parameters and patient survival were assessed in a subset of 445 HNSCC patients with available data using the platform cBioPortal (http://cbioportal.org/) [15].

\section{Statistical analysis}

Chi-squared and Fisher's exact tests were used for comparison between categorical variables. For time-to-event analysis, Kaplan-Meier curves were plotted. Differences between survival times were analyzed by the log-rank method. Cox proportional hazards models were utilized for univariate and multivariate analysis. The hazard ratios 
(HR) with $95 \%$ confidence interval $(\mathrm{Cl})$ and $P$ values were reported. All tests were twosided. $P$ values of $\leq 0.05$ were considered statistically significant.

\section{RESULTS}

\section{Patient characteristics}

The main clinical and pathological features are summarized in Supplementary Table S1. Only 16 patients were women, and the mean age was 59 years (range 30 to 86 years). 360 patients were habitual tobacco smokers, 198 moderate (1-50 pack-year) and 162 heavy (> 50 pack-year), and 341 were alcohol drinkers. Twenty tumors were stage I, 24 stage II, 64 stage III, and 264 stage IV. The series included 147 well, 148 moderately and 76 poorly differentiated tumors. $230(62 \%)$ of 372 patients received postoperative radiotherapy.

The mean and median follow-up times were 34.66 and 21.5 months, respectively, for the whole series. Tumor recurrence was found in $224(60.2 \%)$ cases. The mean and median follow-up times were respectively 71 and 67 months for the patients without recurrence, and 18 and 13.5 months for the patients died of tumor. The five-year disease-specific (DSS) and overall survival (OS) rates were $39 \%$ and $29.7 \%$, respectively.

\section{NOTCH1 and HES1 expression in normal epithelia and HNSCC specimens}

In normal laryngeal and pharyngeal epithelium, NOTCH1 showed a weak cytoplasmic and nuclear staining in basal and suprabasal cell layers, with absence of expression in the most superficial layers (Figure 1A). HES1 expression was also detected in normal epithelium, with a nuclear staining pattern in suprabasal cell layers (Figure 1G).

Membranous NOTCH1 expression was successfully evaluated in 324 out of 372 tumor samples. Only cases with adequate tumor tissue integrity and/or representability were considered, and the staining was scored. Thus, 127 tumors (39\%) showed negative expression (IRS=0), 68 (21\%) low expression (IRS=1), 82 (25\%) moderate expression 
(IRS=2), and 47 (15\%) strong expression (IRS=4). Membranous NOTCH1 expression was also concomitantly accompanied by cytoplasmic staining in some cases. Representative examples of $\mathrm{NOTCH} 1$ staining are shown in Figure 1B-E. Nuclear $\mathrm{NOTCH} 1$ expression was positive in $91(28 \%)$ of the 324 evaluable tumor samples (Figure 1F).

There was a significant positive correlation between nuclear and membranous NOTCH1 expression (Spearman's Rho $=0.502, P<0.001$ ). All the cases with positive nuclear staining showed some degree of membranous staining.

Immunohistochemical analysis of HES1 expression in tumor samples showed a nuclear pattern, with negative expression $(\mathrm{IRS}=0)$ in 110 tumors $(33 \%)$, low expression $(\mathrm{IRS}=1)$ in 81 (24\%), moderate expression (IRS=2) in 86 (26\%), high expression (IRS=4) in 57 (17\%), and 38 non-evaluable cases (Figure 1G-I).

We found that membranous and nuclear $\mathrm{NOTCH} 1$ expression were both significantly correlated with nuclear HES1 expression (Spearman's Rho=0.259, $P<0.001$, and Spearman's Rho=0.241, $P<0.001$, respectively; Table 1).

In addition, the expression of two additional NOTCH1 targets that are key cell cycle regulators, Cyclin D1 and p21, was evaluated by immunohistochemistry and correlated with NOTCH1 expression. Cyclin D1 expression was succesfully evaluated in 360 tumor samples, being positive in 258 (72\%) of them. Positive p21 expression was detected in 204 (63\%) out of 324 evaluable tumor samples (supplementary Figure S1). Membranous and nuclear NOTCH1 expression were both significantly correlated with p21 expression, but not with Cyclin D1 expression (Table 1).

On the other hand, in silico data set analysis of the TCGA HNSCC cohort $(n=520)$ showed that NOTCH1 mRNA levels significantly increased in primary tumors compared to normal tissue samples $(P=0.01$ ) (supplementary Figure $S 2 A)$, whereas HES1 mRNA levels were similar in tumors and the corresponding normal counterparts $(P=$ 0.94) (supplementary Figure S2B). As observed at protein level, NOTCH1 and HES1 
mRNA levels were positively correlated in the TCGA cohort (Spearman's Rho=0.182, $P<0.001)$

Correlations of NOTCH1 and HES1 expression with clinicopathological parameters The expression of both nuclear and membranous NOTCH1 was significantly associated with early stages $(\mathrm{I}-\mathrm{II})(P=0.022$ and $P=0.007$, respectively; Table 2$)$. Nuclear HES1 expression was associated with early T-classification, and with a hypopharyngeal primary site $(P=0.042$ and $P=0.001$, respectively; Table 2$)$. No other significant correlations of $\mathrm{NOTCH} 1$ and HES1 expression with clinicopathological parameters were observed (Table 2).

Similarly, analysis of the TCGA HNSCC dataset $(\mathrm{N}=520)$ further confirmed that NOTCH1 mRNA levels were also significantly higher in early disease stages I-II (unpaired t test, $P=0.03$ ) (supplementary Figure S2C), while HES1 mRNA levels did not show a significant association with stage (unpaired t test, $P=0.06$ ) (supplementary Figure S2D).

Relationship of NOTCH1 and HES1 expression with tumor recurrence and patient survival

A significant inverse correlation was found between membranous NOTCH1 expression and tumor recurrence $(P=0.002$; Table 2). Similarly, nuclear NOTCH1 expression was also more frequent in non-recurrent tumors, although the differences were not significant $(P=0.204 ;$ Table 2$)$. No correlation between HES1 expression and tumor recurrence was observed $(P=1$; Table 2$)$.

Patients harboring either membranous or nuclear NOTCH1-positive tumors concordantly showed significantly improved disease-specific survival (DSS) $(P<0.001$ and $P=0.004$, respectively) and overall survival (OS) $(P<0.001$ and $P=0.005$, 
respectively) (Figure $2 A-D$ ). Notably, significant differences in survival rates were also observed among the different levels of membranous NOTCH1 expression in the tumors, with higher IRS scores showing better survival rates (supplementary Figure S3). Moreover, membranous NOTCH1 expression was associated with better DSS and OS irrespective of the tumor location. Differences were not statistically significant for the laryngeal subgroup of patients, probably due to an insufficient number of cases (Table 3). By contrast, HES1 expression did not correlate with DSS nor OS ( $P=0.63$ and $P=0.67$, respectively) (supplementary Figure S4A-B). p21-positive cases also showed better DSS although the differences did not reach statistical significance $(P=0.058)$ (supplementary Figure S4C-D), whereas Cyclin D1 expression was not associated with survival ( $P=0.5$ and $P=0.62$ for DSS and OS, respectively) (supplementary Figure S4E-F). Correlations between NOTCH1 and HES1 mRNA levels and the patients' survival were also further assessed using the TCGA HNSCC data. Consistent with our IHC protein data, high NOTCH1 mRNA levels were found to associate with a better survival almost reaching significance $(P=0.062)$ (supplementary Figure S5A), while HES1 mRNA levels showed no impact on patients' survival $(P=$ 0.56) (supplementary Figure S5B).

In an attempt to further investigate the impact of NOTCH signaling activation on patient prognosis, the effect of combined expression of membranous NOTCH1 and HES1 on survival was explored. We observed that double-positive cases (NOTCH1+/HES1+) clearly exhibited a significantly improved DSS $(P=0.002)$ and OS $(P=0.03)$, with single positive cases (NOTCH1+/HES1-, NOTCH1-/HES1+) and double-negative cases (NOTCH1-/HES1-) having similar survival rates (Figure 3A-B).

Furthermore, when examining the combined effect of membranous NOTCH1, HES1 and p21 expression, we found that only the triple positive cases (NOTCH1+/HES1+/p21+) were significantly associated with better DSS $(P<0.001)$ and OS $(P=0.004)$ (Figure 3C-D). These findings reinforce that NOTCH pathway activation correlates with a better prognosis in HNSCC patients. 
Multivariate Cox analysis, including tumor localization, T classification, $\mathrm{N}$ classification, degree of differentiation, membranous $\mathrm{NOTCH} 1$ expression, and nuclear NOTCH1 expression showed that the parameters independently associated with a worse DSS were T4 classification, $\mathrm{N}+$ classification and poorly differentiation (Table 4), whereas membranous NOTCH1 expression was an independent predictor of better DSS (HR=0.554; $95 \%$ IC $0.412-0.745 ; \quad P<0.001$; Table 4). Similarly, T4 and $N_{+}$ classifications, and a pharyngeal location of the tumor were independently associated with a worse OS, and membranous NOTCH1 expression with a better OS (HR=0.640; 95\% Cl 0.491-0.835; $P=0.001 ;$ Table 4).

\section{DISCUSSION}

There are evidences of both oncogenic and tumor suppressor roles for NOTCH signaling in different cancers. NOTCH activity has been involved in tumor progression by activating transcription factors promoting cell survival, motility and angiogenesis $[16,17]$. Moreover, activation of the NOTCH pathway regulates the expression of target genes, such as HES1, which has been implicated in stemness, metastasis and multidrug resistance [18]. Several other NOTCH targets are well-known for their relevant roles in tumorigenesis, such as Cyclin D1, с-MYC, and NF-кB [16]. Nevertheless, besides this pro-tumorigenic activity of NOTCH signaling, increasing evidence has also pointed out to a tumor suppressive role for this pathway. Thus, deletions and inactivating mutations in various NOTCH family members are frequently and commonly detected in a variety of tumor types [19]. Specifically, loss-of-function mutations in NOTCH1 have been reported in HNSCC, cutaneous and lung squamous cell carcinomas, suggesting that NOTCH1 may act as a tumor suppressor of squamous cell carcinogenesis $[8,9,20,21]$. Moreover, a recent report has demonstrated that recurrent but infrequent driver mutations found in HNSCC converge onto the NOTCH 
signaling pathway, resulting in inactivation of $\mathrm{NOTCH}$ signaling in $67 \%$ of patients, thus emerging as one of the most commonly dysregulated pathways in HNSCC [22].

Our results also evidence this conflicting role of NOTCH in HNSCC carcinogenesis. We have found higher levels of $\mathrm{NOTCH} 1$ protein expression in tumors than in the normal epithelium, and these data were confirmed at the NOTCH1 mRNA expression level in the TCGA database analysis, suggesting an oncogenic role for NOTCH1. Contrary to this, our results also show a lower $\mathrm{NOTCH} 1$ expresion in advanced tumor stages, suggesting that NOTCH1 expresion/activity decreases along disease progression, therefore suggesting a tumor suppressor role for this protein. Hence, increased expression of $\mathrm{NOTCH} 1$ in early stages of the disease may represent an attempt to impair malignant progression, as suggested in other solid tumors [19].

In HNSCC, the expression of NOTCH pathway components has only been examined in small patient cohorts (mostly in Asian populations) with differing results [5,10]. Some studies implicated $\mathrm{NOTCH} 1$ as pro-oncogenic, and described the association of NOTCH1 expression with poor prognosis [23,24], lymph node metastasis [25], poor differentiation and resistance to chemotherapy [26]. In contrast, other studies correlated high NOTCH1 expression with improved survival in oropharyngeal squamous cell carcinoma patients [27] and HNSCC patients [28]. Our results show that the expression of $\mathrm{NOTCH} 1$ is independently associated with better disease control and survival rates. In addition, NOTCH1 mRNA expression analyses in the TCGA database also shows a tendency for a better survival in patients with elevated NOTCH1 mRNA levels. Interestingly, most of the reports that correlate NOTCH1 expression with poor prognosis are focused on subsets of Asian patients, whereas the association of $\mathrm{NOTCH} 1$ expression with better prognosis has been reported in studies that included Caucasian patients $[10,27,28]$. In the same way, the studies that found inactivating mutations of $\mathrm{NOTCH} 1$ included mostly Caucasian patients [7,8], whereas studies including Asian patients have shown that more than half of the NOTCH1 mutations are 
activating ones $[29,30]$. These findings suggest that $\mathrm{NOTCH} 1$ promotes distinct tumorigenic mechanisms in patients from different ethnical populations.

HES1 is one of the few known gene targets indicating NOTCH pathway activation [1]. As expected, we have found a close correlation between $\mathrm{NOTCH} 1$ and HES1 expression. However, HES1 expression in tumor samples was slightly more frequent than NOTCH1 expression. Interestingly, although HES1 expression alone was not related with the prognosis of HNSCC patients, only the cases that were $\mathrm{NOTCH} 1+/ \mathrm{HES} 1+$ have a better survival. As these cases probably represent the cases with true NOTCH pathway activation, this reinforces that the activation of the NOTCH pathway is in fact associated with a better prognosis.

We have also found that HES1 expression is present in normal epithelium, and the analysis of the TCGA shows no statistically significant difference in HES1 expression between HNSCC and control tissues, suggesting that NOTCH pathway is activated in normal epithelium. This activation of $\mathrm{NOTCH}$ pathway in normal epithelium is wellknown in the epidermis and associated with differentiation, supporting the tumor suppressor role of this pathway in the squamous epithelia [31]. Few other studies have analyzed the expression of HES1 in HNSCC. A comprehensive study of $\mathrm{NOTCH}$ signaling pathway status reported overexpression of HES1 and/or HEY1 in $30 \%$ of HNSCC samples, which was correlated with NOTCH1 overexpression, and significantly lower HES1/HEY1 expression in tumors with inactivating NOTCH1 mutations [6]. This study proposes a bimodal pattern of $\mathrm{NOTCH}$ pathway alterations in HNSCC, with a subset of tumors harboring inactivating $\mathrm{NOTCH} 1$ receptor mutations while a larger subset exhibits other NOTCH1 pathway alterations, such as increased HES1 and/or HEY1 expression driving downstream pathway activation. However, this study did not address the prognostic significance of NOTCH pathway activation. Another study also found HES1 overexpression in oral squamous cell carcinomas but was not correlated with the expression of NOTCH intracellular domain (NICD) [32]. In that study, HES1 expression was associated with a poorer prognosis, but only when associated with c- 
MYC-positive expression [32]. Taking together these studies and our results suggest that $\mathrm{NOTCH}$ pathway is activated in normal epithelium and remain activated in a subset of tumors, depending the clinical significance on the genetic background of the individual tumors.

In this way, one of the mechanisms by which $\mathrm{NOTCH}$ signaling may act in an antitumorigenic manner include the induction of p21, which promotes cell cycle arrest $[19,33]$. In agreement with this hypothesis, we found a positive correlation between NOTCH1 and p21 expression in our HNSCC cohort, as described in other tumor types [34,35]. Given the anti-proliferative function of p21 [33], this finding could provide a plausible explanation for the better prognosis observed in NOTCH1-positive patients. In fact, a better survival was observed in the subset of patients with simultaneous $\mathrm{NOTCH} 1+/ \mathrm{HES} 1+/ \mathrm{p} 21+$ expression. In contrast, NOTCH1 expression was not correlated in our series with Cyclin D1 expression, which favors cell cycle progression. NOTCH1 signaling has been associated with either increased $[36,37]$ or reduced [38] Cyclin D1 in different tumor types, suggesting that the regulation of Cyclin D1 expression by NOTCH signaling may depend on specific cell/tissue contexts.

In summary, this study represents the most comprehensive analysis and the largest HNSCC cohort reported to date directed at establishing the clinical and prognostic relevance of $\mathrm{NOTCH} 1$ signaling activation in $\mathrm{HNSCC}$, thereby bringing together valuable data to improve patient stratification and guide treatment based on the expression of various $\mathrm{NOTCH} 1$ signaling components. Patients carrying high expression of $\mathrm{NOTCH} 1$ showed a reduced relapse risk and significantly improved prognosis. In particular, the subset of patients harboring $\mathrm{NOTCH} 1+/ \mathrm{HES} 1+/ \mathrm{p} 21+$ tumors exhibited the highest survival rates. Inhibitors of NOTCH signaling are already in clinical testing in other malignancies such as breast, ovarian, pancreatic and smallcell lung cancers $[39,40]$. However, according to our findings, NOTCH inhibition seems less promising in HNSCC, since patients with high NOTCH1 expression clearly and consistently demonstrated a better survival in our study. These findings should 
therefore be highly relevant for clinical evaluation of $\mathrm{NOTCH}$-targeting therapies in HNSCC patients.

\section{ACKNOWLEDGMENTS}

We thank the samples and technical assistance kindly provided by the Principado de Asturias BioBank (PT13/0010/0046), financed jointly by Servicio de Salud del Principado de Asturias, Instituto de Salud Carlos III and Fundación Bancaria Cajastur and integrated in the Spanish National Biobanks Network. We also thank Juan Pérez Ortega for his excellent administrative support.

\section{FINANCIAL SUPPORT}

This study was supported by grants from the Plan Nacional de I+D+l 2013-2016 [ISCIII (PI16/00280 and PI19/00560 to JMGP), CIBERONC (CB16/12/00390 to JPR and CB16/12/00295 to AC), Asociación Española contra el Cáncer (AECC; 2019/ INVES19001ALVA to MAF), the Instituto de Investigación Sanitaria del Principado de Asturias (ISPA), Ayudas a Grupos PCTI Principado de Asturias (IDI2018/155 to JPR), Fundación Bancaria Caja de Ahorros de Asturias-IUOPA and the FEDER Funding Program from the European Union.

\section{REFERENCES}

[1] Ntziachristos P, Lim JS, Sage J, Aifantis I. From fly wings to targeted cancer therapies: a centennial for notch signaling. Cancer Cell. 2014;25:318-34. doi:10.1016/j.ccr.2014.02.018

[2] Fukusumi T, Califano JA. The NOTCH Pathway in Head and Neck Squamous Cell Carcinoma. J Dent Res. 2018;97:645-3. doi:10.1177/00220345187602975.

[3] Artavanis-Tsakonas S, Rand MD, Lake RJ. Notch signaling: cell fate control and signal integration in development. Science. 1999;284:770-6. 
[4] Porcheri C, Meisel CT, Mitsiadis T. Multifactorial Contribution of Notch Signaling in Head and Neck Squamous Cell Carcinoma. Int J Mol Sci. 2019;20:1520. doi:10.3390/ijms20061520

[5] Nyman PE, Buehler D, Lambert PF. Loss of Function of Canonical Notch Signaling Drives Head and Neck Carcinogenesis. Clin Cancer Res. 2018;24:6308-18. doi:10.1158/1078-0432.CCR-17-3535

[6] Sun W, Gaykalova DA, Ochs MF, Mambo E, Arnaoutakis D, Liu Y, et al. Activation of the NOTCH pathway in head and neck cancer. Cancer Res. 2014;74:1091-104. doi: 10.1158/0008-5472.CAN-13-1259.

[7] Agrawal N, Frederick MJ, Pickering CR, Bettegowda C, Chang K, Li RJ, et al. Exome sequencing of head and neck squamous cell carcinoma reveals inactivating mutations in NOTCH1. Science. 2011;333:1154-7. doi: 10.1126/science.1206923. [8] Stransky N, Egloff AM, Tward AD, Kostic AD, Cibulskis K, Sivachenko A, et al. The mutational landscape of head and neck squamous cell carcinoma. Science. 2011;333:1157-60. doi: 10.1126/science.1208130.

[9] Pickering CR, Zhang J, Yoo SY, Bengtsson L, Moorthy S, Neskey DM, et al. Integrative genomic characterization of oral squamous cell carcinoma identifies frequent somatic drivers. Cancer Discov. 2013;3:770-81. doi: 10.1158/2159-8290.CD12-0537.

[10] Zhao YY, Yu GT, Xiao T, Hu J. The Notch signaling pathway in head and neck squamous cell carcinoma: A meta-analysis. Adv Clin Exp Med. 2017;26:881-7. doi:10.17219/acem/64000

[11] Rodrigo JP, Heideman DA, García-Pedrero JM, Fresno MF, Brakenhoff RH, Díaz Molina JP, et al. Time trends in the prevalence of HPV in oropharyngeal squamous cell carcinomas in northern Spain (1990-2009). Int J Cancer 2014;134:487-92. doi: 10.1002/ijc.28355.

[12] Rodrigo JP, Hermsen MA, Fresno MF, Brakenhoff RH, García-Velasco F, Snijders PJ, et al. Prevalence of human papillomavirus in laryngeal and hypopharyngeal 
squamous cell carcinomas in northern Spain. Cancer Epidemiol 2015;39:37-41. doi: 10.1016/j.canep.2014.11.003.

[13] Cancer Genome Atlas Network Comprehensive genomic characterization of head and neck squamous cell carcinomas. Nature 2015;517:576-82. doi:

10.1038/nature14129.

[14] Chandrashekar DS, Bashel B, Balasubramanya SAH, Creighton CJ, Rodriguez IP, Chakravarthi BVSK, et al. UALCAN: A portal for facilitating tumor subgroup gene expression and survival analyses. Neoplasia 2017;19:649-58. doi:

10.1016/j.neo.2017.05.002.

[15] Cerami E, Gao J, Dogrusoz U, Gross BE, Sumer SO, Aksoy BA, et al. The cBio cancer genomics portal: an open platform for exploring multidimensional cancer genomics data. Cancer Discov 2012;2:401-4. doi: 10.1158/2159-8290.CD-12-0095. [16] Wang Z, Li Y, Banerjee S, Sarkar FH. Emerging role of Notch in stem cells and cancer. Cancer Lett 2009;279:8-12. doi:10.1016/j.canlet.2008.09.030

[17] Ranganathan P, Weaver KL, Capobianco AJ. Notch signalling in solid tumours: a little bit of everything but not all the time. Nat Rev Cancer 2011;11:338-51. doi:10.1038/nrc3035

[18] Liu ZH, Dai XM, Du B. Hes1: a key role in stemness, metastasis and multidrug resistance. Cancer Biol Ther 2015;16:353-9. doi:10.1080/15384047.2015.1016662 [19] Nowell CS, Radtke F. Notch as a tumour suppressor. Nat Rev Cancer 2017;17:145-59. doi:10.1038/nrc.2016.145

[20] South AP, Purdie KJ, Watt SA, Haldenby S, den Breems N, Dimon M, et al. $\mathrm{NOTCH} 1$ mutations occur early during cutaneous squamous cell carcinogenesis. J Invest Dermatol 2014;134:2630-8. doi: 10.1038/jid.2014.154 [21] Wang NJ, Sanborn Z, Arnett KL, Bayston LJ, Liao W, Proby CM, et al. Loss-offunction mutations in Notch receptors in cutaneous and lung squamous cell carcinoma. Proc Natl Acad Sci USA 2011;108: 17761-6. doi: 10.1073/pnas.1114669108. 
[22] Loganathan SK, Schleicher K, Malik A, Quevedo R, Langille E, Teng K, et al. Rare driver mutations in head and neck squamous cell carcinomas converge on NOTCH signaling. Science 2020;367:1264-9. doi: 10.1126/science.aax0902.

[23] Lee SH, Do SI, Lee HJ, Kang HJ, Koo BS, Lim YC. Notch1 signaling contributes to stemness in head and neck squamous cell carcinoma. Lab Invest 2016;96:508-16. doi:10.1038/labinvest.2015.163

[24] Lin JT, Chen MK, Yeh KT, Chang CS, Chang TH, Lin CY, et al. Association of high levels of Jagged-1 and Notch-1 expression with poor prognosis in head and neck cancer. Ann Surg Oncol 2010;17:2976-83. doi: 10.1245/s10434-010-1118-9. [25] Zhang TH, Liu HC, Zhu LJ, Chu M, Liang YJ, Liang LZ, et al. Activation of Notch signaling in human tongue carcinoma. J Oral Pathol Med 2011;40:37-45. doi: 10.1111/j.1600-0714.2010.00931.x.

[26] Zhao ZL, Zhang L, Huang CF, Ma SR, Bu LL, Liu JF, et al. NOTCH1 inhibition enhances the efficacy of conventional chemotherapeutic agents by targeting head neck cancer stem cell. Sci Rep 2016;6:24704. doi: 10.1038/srep24704.

[27] Kaka AS, Nowacki NB, Kumar B, Zhao S, Old MO, Agrawal A, et al. Notch1 overexpression correlates to improved survival in cancer of the oropharynx.

Otolaryngol Head Neck Surg 2017 ;156:652-9. doi: 10.1177/0194599816688178. [28] Wirth M, Jira D, Ott A, Piontek G, Pickhard A. High NOTCH1 mRNA expression is associated with better survival in HNSCC. Int J Mol Sci 2018;19:830.

doi:10.3390/ijms 19030830

[29] Song X, Xia R, Li J, Long Z, Ren H, Chen W, et al. Common and complex Notch1 mutations in Chinese oral squamous cell carcinoma. Clin Cancer Res 2014;20:701-10. doi: 10.1158/1078-0432.CCR-13-1050.

[30] Izumchenko E, Sun K, Jones S, Brait M, Agrawal N, Koch W, et al. Notch1 mutations are drivers of oral tumorigenesis. Cancer Prev Res (Phila) 2015;8:277-86. doi: 10.1158/1940-6207.CAPR-14-0257. 
[31] Panelos J, Massi D. Emerging role of Notch signaling in epidermal differentiation and skin cancer. Cancer Biol Ther 2009;8:1986-93. doi: 10.4161/cbt.8.21.992.

[32] Gokulan R, Halagowder D. Expression pattern of Notch intracellular domain (NICD) and Hes-1 in preneoplastic and neoplastic human oral squamous epithelium: their correlation with c-Myc, clinicopathological factors and prognosis in Oral cancer. Med Oncol 2014;31:126. doi:10.1007/s12032-014-0126-1.

[33] Devgan V, Mammucari C, Millar SE, Brisken C, Dotto GP. p21WAF1/Cip1 is a negative transcriptional regulator of Wnt4 expression downstream of Notch1 activation. Genes Dev 2005;19:1485-95. doi:10.1101/gad.341405

[34] Kunnimalaiyaan M, Vaccaro AM, Ndiaye MA, Chen H. Overexpression of the $\mathrm{NOTCH} 1$ intracellular domain inhibits cell proliferation and alters the neuroendocrine phenotype of medullary thyroid cancer cells. J Biol Chem 2006;281:39819-30. doi:10.1074/jbc.M603578200.

[35] Qi R, An H, Yu Y, Zhang M, Liu S, Xu H, et al. Notch1 signaling inhibits growth of human hepatocellular carcinoma through induction of cell cycle arrest and apoptosis. Cancer Res 2003;63:8323-9.

[36] Cohen B, Shimizu M, Izrailit J, Ng NF, Buchman Y, Pan JG, et al. Cyclin D1 is a direct target of JAG1-mediated Notch signaling in breast cancer. Breast Cancer Res Treat 2010;123:113-24. doi:10.1007/s10549-009-0621-9.

[37] Ling H, Sylvestre JR, Jolicoeur P. Notch1-induced mammary tumor development is cyclin D1-dependent and correlates with expansion of pre-malignant multipotent ductlimited progenitors. Oncogene 2010;29:4543-54. doi:10.1038/onc.2010.186 [38] Sui C, Zhuang C, Sun D, Yang L, Zhang L, Song L. Notch1 regulates the JNK signaling pathway and increases apoptosis in hepatocellular carcinoma. Oncotarget 2017;8:45837-47. doi:10.18632/oncotarget.17434

[39] Yen WC, Fischer MM, Axelrod F, Bond C, Cain J, Cancilla B, et al. Targeting Notch signaling with a Notch2/Notch3 antagonist (tarextumab) inhibits tumor growth 
and decreases tumor-initiating cell frequency. Clin Cancer Res 2015;21:2084-95. doi: 10.1158/1078-0432.CCR-14-2808.

[40] Takebe N, Miele L, Harris PJ, Jeong W, Bando H, Kahn M, et al. Targeting Notch, Hedgehog, and Wnt pathways in cancer stem cells: clinical update. Nat Rev Clin Oncol 2015;12:445-64. doi: 10.1038/nrclinonc.2015.61. 


\section{FIGURE LEGENDS}

Figure 1. Immunohistochemical analysis of $\mathrm{NOTCH} 1$ and $\mathrm{HES} 1$ expression in HNSCC. Representative images of NOTCH1 expression in normal epithelium (A), a tumor showing negative NOTCH1 expression (B), examples of tumors with low (C), moderate (D), or strong membranous $\mathrm{NOTCH} 1$ expression $(\mathrm{E})$, and a tumor with nuclear NOTCH1 expression (F), and examples of HES1 expression in normal epithelium (G), and tumors with negative $(H)$, and positive nuclear HES1 expression (I). Scale bars $=100 \mu \mathrm{m}$.

Figure 2. Kaplan-Meier disease specific (DSS) and overall survival (OS) curves according to the expression of membranous $\mathrm{NOTCH}_{1}(\mathrm{~A}, \mathrm{~B})$ and nuclear $\mathrm{NOTCH} 1$ (C, D). $P$ values were estimated using the Log-rank test.

Figure 3. Kaplan-Meier disease specific (DSS) and overall survival (OS) curves according to the combined expression of NOTCH1 and HES1 proteins (A, B), and the combined expression of $\mathrm{NOTCH} 1, \mathrm{HES} 1$ and $\mathrm{p} 21$ proteins $(\mathrm{C}, \mathrm{D}) . P$ values were estimated using the Log-rank test. 


\section{Electronic Supplementary Material}

Supplementary material NOTCH.pdf 
Table 1. Correlations between the expression of NOTCH1 and the target genes HES1, p21 and Cyclin D1.

\begin{tabular}{|c|c|c|c|c|c|c|c|c|c|}
\hline \multirow[b]{2}{*}{$\begin{array}{l}\text { NOTCH1 } \\
\text { expression }\end{array}$} & \multicolumn{3}{|c|}{ HES1 expression } & \multicolumn{3}{|c|}{ p21 expression } & \multicolumn{3}{|c|}{ Cyclin D1 expression } \\
\hline & Negative & Positive & $P$ & Negative & Positive & $P$ & Negative & Positive & $\boldsymbol{P}$ \\
\hline $\begin{array}{l}\text { Membranous: } \\
\text { - Negative }\end{array}$ & $\begin{array}{c}58 \\
(47.5 \%)\end{array}$ & $\begin{array}{c}64 \\
(52.5 \%)\end{array}$ & $<0.001$ & $\begin{array}{c}55 \\
(45 \%)\end{array}$ & $\begin{array}{c}67 \\
(55 \%)\end{array}$ & 0.030 & $\begin{array}{c}41 \\
(32 \%)\end{array}$ & $\begin{array}{c}86 \\
(68 \%)\end{array}$ & 0.21 \\
\hline - Positive & $\begin{array}{c}44 \\
(22.6 \%)\end{array}$ & $\begin{array}{c}150 \\
(77.4 \%)\end{array}$ & & $\begin{array}{c}59 \\
(32.5 \%)\end{array}$ & $\begin{array}{c}122 \\
(67.5 \%)\end{array}$ & & $\begin{array}{c}50 \\
(26 \%)\end{array}$ & $\begin{array}{c}144 \\
(74 \%)\end{array}$ & \\
\hline $\begin{array}{l}\text { Nuclear: } \\
\text { - Negative }\end{array}$ & $\begin{array}{c}89 \\
(39 \%)\end{array}$ & $\begin{array}{c}137 \\
(61 \%)\end{array}$ & $<0.001$ & $\begin{array}{c}90 \\
(41 \%)\end{array}$ & $\begin{array}{c}128 \\
(59 \%)\end{array}$ & 0.036 & $\begin{array}{c}72 \\
(31 \%)\end{array}$ & $\begin{array}{c}159 \\
(69 \%)\end{array}$ & 0.075 \\
\hline - Positive & $\begin{array}{c}13 \\
(14 \%)\end{array}$ & $\begin{array}{c}77 \\
(86 \%)\end{array}$ & & $\begin{array}{c}24 \\
(28 \%)\end{array}$ & $\begin{array}{c}61 \\
(72 \%)\end{array}$ & & $\begin{array}{c}19 \\
(21 \%)\end{array}$ & $\begin{array}{c}71 \\
(79 \%)\end{array}$ & \\
\hline Total & 102 & 214 & & 114 & 189 & & 91 & 230 & \\
\hline
\end{tabular}


Table 2. Associations of NOTCH1 and HES1 expression with clinicopathological features.

\begin{tabular}{|c|c|c|c|c|c|c|c|c|}
\hline Characteristic & $\begin{array}{l}\text { No. } \\
\text { Cases for } \\
\text { NOTCH1 }\end{array}$ & $\begin{array}{c}\text { Membranous } \\
\text { NOTCH1 } \\
\text { Expression } \\
(\%) \\
\end{array}$ & $\boldsymbol{P}$ & $\begin{array}{c}\text { Nuclear } \\
\text { NOTCH1 } \\
\text { Expression } \\
(\%) \\
\end{array}$ & $\boldsymbol{P}$ & $\begin{array}{l}\text { No. } \\
\text { Cases } \\
\text { for } \\
\text { HES1 }\end{array}$ & $\begin{array}{c}\text { Nuclear } \\
\text { HES1 } \\
\text { Expression } \\
(\%) \\
\end{array}$ & $P$ \\
\hline \multicolumn{9}{|l|}{ Location } \\
\hline Oropharynx & 216 & $124(57)$ & \multirow{3}{*}{$0.175^{\#}$} & $63(29)$ & \multirow{3}{*}{$0.575^{\#}$} & 229 & $143(62)$ & \multirow{3}{*}{$0.001^{\#}$} \\
\hline Hypopharynx & 54 & $35(65)$ & & $12(22)$ & & 52 & $46(88)$ & \\
\hline Larynx & 54 & $38(70)$ & & $16(30)$ & & 53 & $35(66)$ & \\
\hline \multicolumn{9}{|l|}{ pT Classification } \\
\hline T1-T2 & 95 & $66(70)$ & \multirow{3}{*}{$0.098^{\#}$} & $32(34)$ & \multirow{3}{*}{$0.183^{\#}$} & 97 & $77(75)$ & \multirow{3}{*}{$0.042^{\#}$} \\
\hline T3 & 113 & $67(59)$ & & $33(29)$ & & 114 & $81(68)$ & \\
\hline T4 & 116 & $64(55)$ & & $26(22)$ & & 123 & $74(59)$ & \\
\hline \multicolumn{9}{|l|}{ pN Classification } \\
\hline NO & 87 & $61(68)$ & \multirow[t]{2}{*}{$0.202^{\dagger}$} & $30(34)$ & \multirow[t]{2}{*}{$0.127^{\dagger}$} & 85 & $52(61)$ & \multirow[t]{2}{*}{$0.184^{\dagger}$} \\
\hline N1-3 & 237 & $143(59)$ & & $61(26)$ & & 249 & $172(69)$ & \\
\hline \multicolumn{9}{|l|}{ Stage } \\
\hline I-II & 34 & $27(79)$ & \multirow{3}{*}{$0.022^{\#}$} & $17(50)$ & \multirow{3}{*}{$0.007^{\#}$} & 31 & $25(81)$ & \multirow{3}{*}{$0.205^{\#}$} \\
\hline III & 55 & $37(67)$ & & $17(31)$ & & 56 & $35(62)$ & \\
\hline IV & 235 & $133(57)$ & & $57(24)$ & & 247 & $164(66)$ & \\
\hline \multicolumn{9}{|l|}{ Degree of } \\
\hline differentiation & 129 & $70(54)$ & \multirow{3}{*}{$0.128^{\#}$} & $38(25)$ & \multirow{3}{*}{$0.882^{\#}$} & 129 & $87(67)$ & \multirow{3}{*}{$0.780^{\#}$} \\
\hline Well & 130 & $83(64)$ & & $36(28)$ & & 135 & $88(65)$ & \\
\hline $\begin{array}{c}\text { Moderately } \\
\text { Poorly }\end{array}$ & 65 & $44(68)$ & & $17(26)$ & & 70 & $49(70)$ & \\
\hline \multicolumn{9}{|l|}{ Recurrence } \\
\hline No & 121 & $87(72)$ & \multirow[t]{2}{*}{$0.002^{\dagger}$} & $39(32)$ & \multirow[t]{2}{*}{$0.204^{\dagger}$} & 124 & $83(67)$ & \multirow[t]{2}{*}{$1^{\dagger}$} \\
\hline Yes & 203 & $110(54)$ & & $52(26)$ & & 210 & $141(67)$ & \\
\hline Total & 324 & $197(61)$ & & $91(28)$ & & 334 & $224(67)$ & \\
\hline
\end{tabular}


Table 3. Five-year disease-specific survival (DSS) and overall survival (OS) according to membranous NOTCH1 expression.

\begin{tabular}{|l|c|c|c|c|}
\hline \multicolumn{1}{|c|}{ Localization } & $\begin{array}{c}\text { NOTCH1 } \\
\text { positive }\end{array}$ & $\begin{array}{c}\text { NOTCH1 } \\
\text { negative }\end{array}$ & $\begin{array}{c}\text { Hazard ratio } \\
\mathbf{( 9 5 \% ~ C l )}\end{array}$ & $\boldsymbol{P}$ \\
\hline Oropharynx (n=256) & & & & \\
- DSS & $59 \%$ & $33 \%$ & $0.536(0.374-0.766)$ & 0.001 \\
- OS & $54 \%$ & $32 \%$ & $0.631(0.461-0.863)$ & 0.003 \\
\hline Hypopharynx (n=54) & $30 \%$ & $0 \%$ & $0.446(0.228-0.876)$ & 0.019 \\
- DSS & $26 \%$ & $0 \%$ & $0.424(0.224-0.803)$ & 0.008 \\
- OS & $63 \%$ & $35 \%$ & $0.527(0.227-1.223)$ & 0.129 \\
\hline Larynx (n=54) & $53 \%$ & $32 \%$ & $0.612(0.282-1.332)$ & 0.216 \\
- DSS & & & & \\
- OS & & & & \\
\hline
\end{tabular}

DSS: Disease-specific survival; OS: Overall survival; 95\% Cl: 95\% Confidence Interval 
Table 4. Multivariate Cox analysis for disease-specific survival and overall survival.

\begin{tabular}{|c|c|c|c|c|}
\hline \multirow{2}{*}{ Parameter } & \multicolumn{2}{|c|}{ Disease-specific survival } & \multicolumn{2}{|c|}{ Overall survival } \\
\hline & HR (95\% Cl) & $\boldsymbol{P}$ & HR (95\% Cl) & $\boldsymbol{P}$ \\
\hline $\begin{array}{l}\text { Localization } \\
\text { - Larynx } \\
\text { - Oropharynx } \\
\text { - Hypopharynx }\end{array}$ & $\begin{array}{l}1 \\
1.467(0.927-2.322) \\
1.645(0.966-2.802)\end{array}$ & $\begin{array}{l}0.102 \\
0.067\end{array}$ & $\begin{array}{l}1 \\
1.603(1.069-2.404) \\
1.756(1.087-2.838)\end{array}$ & $\begin{array}{l}0.022 \\
0.022\end{array}$ \\
\hline $\begin{array}{l}\text { pT classification } \\
- \text { T1-2 } \\
- \text { T3 } \\
- \text { T4 }\end{array}$ & $\begin{array}{l}1 \\
1.203(0.809-1.789) \\
1.881(1.288-2.749)\end{array}$ & $\begin{array}{l}0.362 \\
0.001\end{array}$ & $\begin{array}{l}1 \\
1.073(0.765-1.504) \\
1.663(1.206-2.292)\end{array}$ & $\begin{array}{l}0.685 \\
0.002\end{array}$ \\
\hline $\begin{array}{l}\text { pN classification } \\
-\mathrm{NO} \\
-\mathrm{N}+\end{array}$ & $\begin{array}{l}1 \\
2.310(1.557-3.426)\end{array}$ & $<0.001$ & $\begin{array}{l}1 \\
1.759(1.272-2.433)\end{array}$ & 0.001 \\
\hline $\begin{array}{l}\text { Degree of differentiation } \\
\text { - Well } \\
\text { - Moderately } \\
\text { - Poorly }\end{array}$ & $\begin{array}{l}1 \\
0.99(0.708-1.384) \\
1.502(1.013-2.226)\end{array}$ & $\begin{array}{l}0.952 \\
0.043\end{array}$ & $\begin{array}{l}1 \\
1.053(0.785-1.414) \\
1.375(0.957-1.976)\end{array}$ & $\begin{array}{l}0.729 \\
0.085\end{array}$ \\
\hline $\begin{array}{l}\text { Membranous NOTCH1 } \\
\text { - Negative } \\
\text { - Positive }\end{array}$ & $\begin{array}{l}1 \\
0.554(0.412-0.745)\end{array}$ & $<0.001$ & $\begin{array}{l}1 \\
0.640(0.491-0.835)\end{array}$ & 0.001 \\
\hline $\begin{array}{l}\text { Nuclear NOTCH1 } \\
\text { - Negative } \\
\text { - Positive }\end{array}$ & $\begin{array}{l}1 \\
1.050(0.697-1.583)\end{array}$ & 0.815 & $\begin{array}{l}1 \\
1.009(0.714-1.426)\end{array}$ & 0.960 \\
\hline
\end{tabular}

HR: Hazard Ratio; 95\% Cl: 95\% Confidence Interval 
Click here to download high resolution image
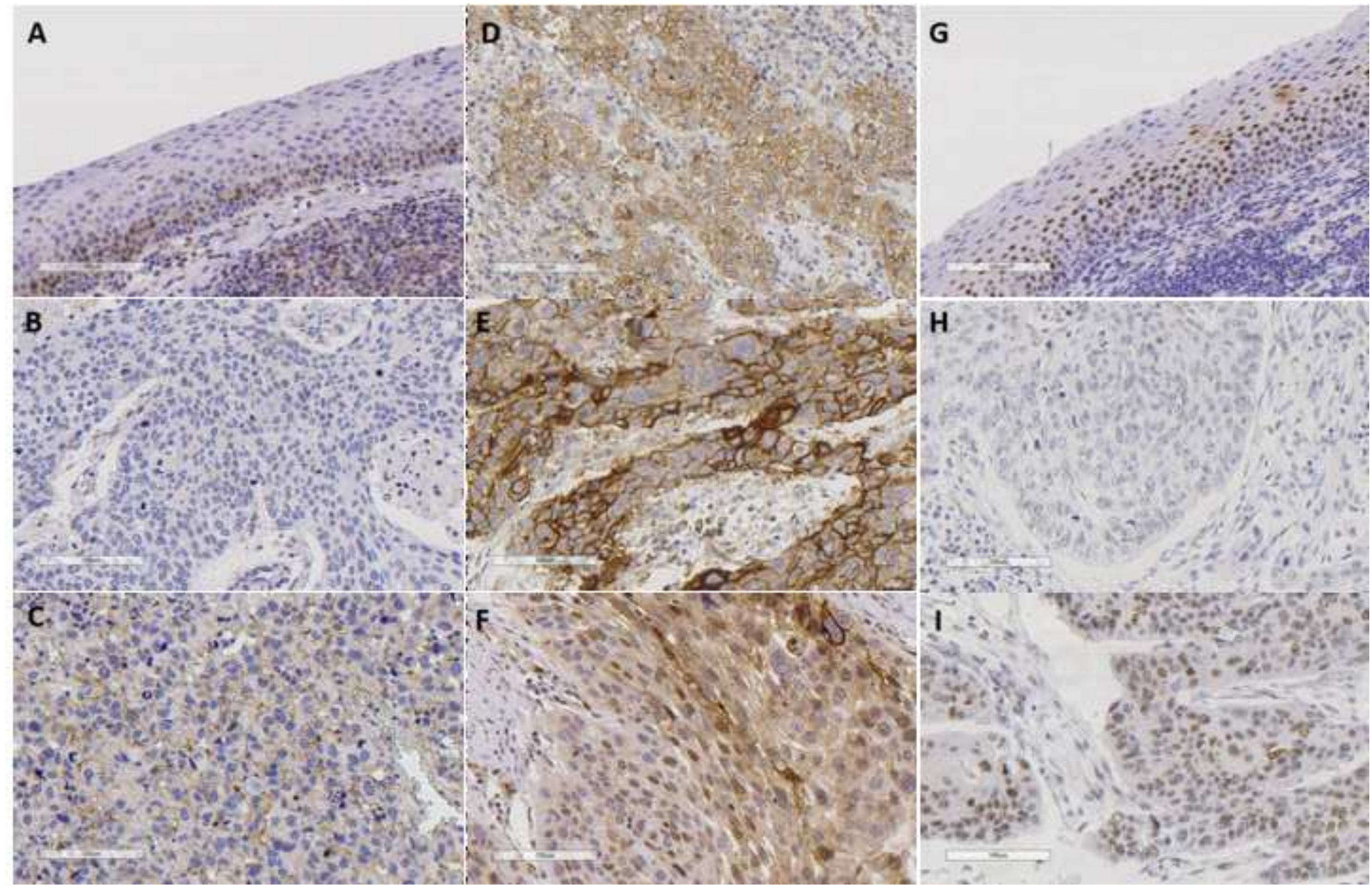

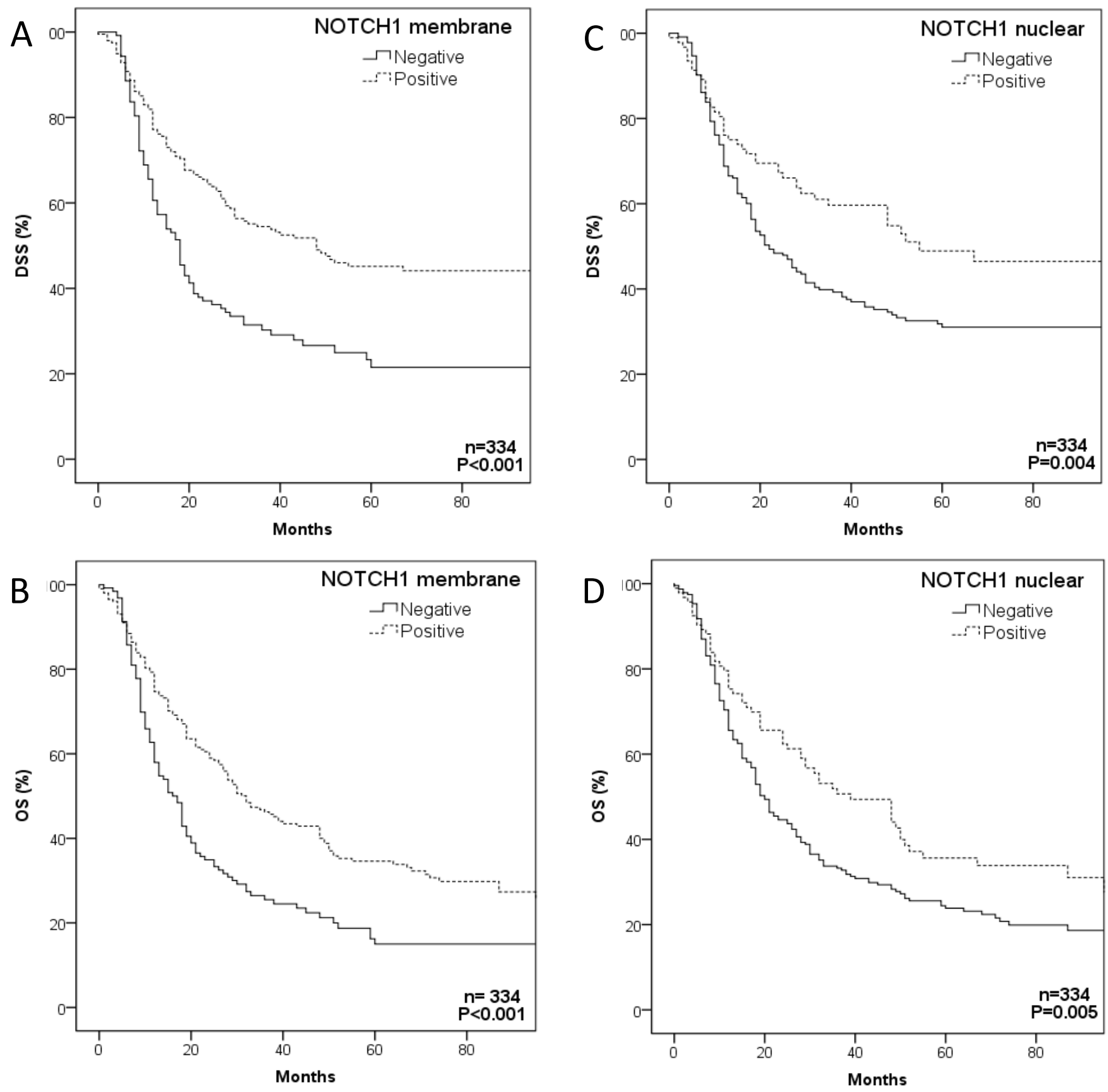

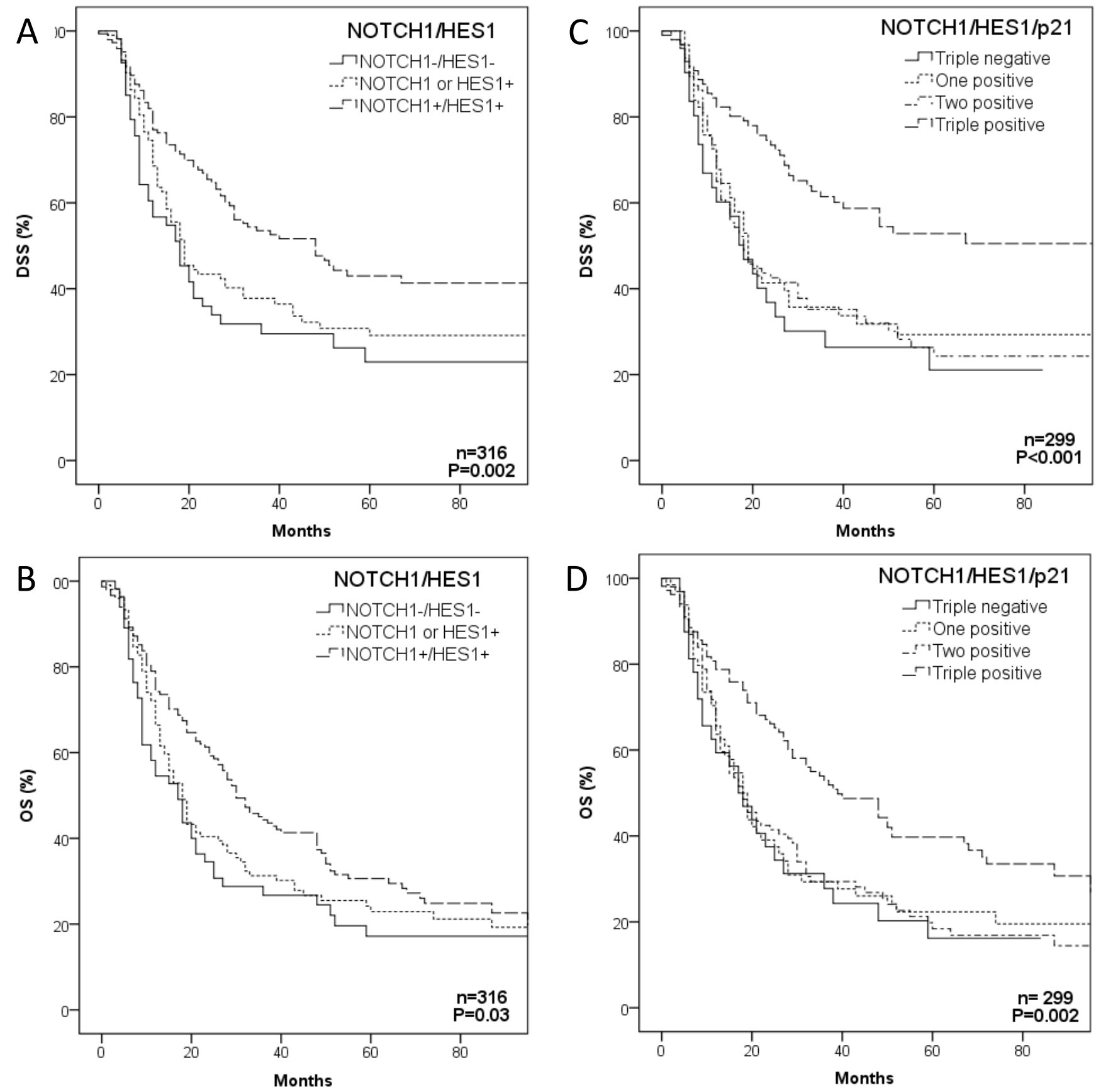

Supplementary Data (online only)
Click here to download Supplementary Data (online only): Supplementary material NOTCH.pdf 